\title{
Comparison of CT colonography, colonoscopy, sigmoidoscopy and faecal occult blood tests for the detection of advanced adenoma in an average risk population
}

\author{
A Graser, ${ }^{1}$ P Stieber, ${ }^{2}$ D Nagel, ${ }^{2}$ C Schäfer, ${ }^{3}$ D Horst ${ }_{1}^{4}$ C R Becker, ${ }^{1} \mathrm{~K}$ Nikolaou, ${ }^{1}$ \\ A Lottes, ${ }^{3}$ S Geisbüsch, ${ }^{1} \mathrm{H}$ Kramer, ${ }^{1}$ A C Wagner, ${ }^{3} \mathrm{H}$ Diepolder, ${ }^{3} \mathrm{~J}$ Schirra, ${ }^{3} \mathrm{H}$ J Roth, \\ D Seidel, ${ }^{2}$ B Göke, ${ }^{3}$ M F Reiser, ${ }^{1}$ F T Kolligs ${ }^{3}$
}

${ }^{1}$ Department of Clinical Radiology, University of Munich, Klinikum Grosshadern, Munich, Germany; ${ }^{2}$ Department of Clinical Chemistry, University of Munich, Klinikum Grosshadern, Munich, Germany; ${ }^{3}$ Department of Medicine II, University of Munich, Klinikum Grosshadern, Munich, Germany; ${ }^{4}$ Department of Pathology, University of Munich, Klinikum Grosshadern, Munich, Germany; ${ }^{5}$ Limbach Laboratory, Heidelberg, Germany

Correspondence to:

Dr A Graser, Department of Clinical Radiology, University of Munich, Marchioninistrasse 15, D-81377 Munich, Germany; anno.graser@med.unimuenchen.de

Revised 28 August 2008 Accepted 1 September 2008 Accepted for Online First 13 October 2008

\section{ABSTRACT}

Background and aims: This prospective trial was designed to compare the performance characteristics of five different screening tests in parallel for the detection of advanced colonic neoplasia: CT colonography (CTC), colonoscopy (OC), flexible sigmoidoscopy (FS), faecal immunochemical stool testing (FIT) and faecal occult blood testing (FOBT).

Methods: Average risk adults provided stool specimens for FOBT and FIT, and underwent same-day low-dose 64multidetector row CTC and OC using segmentally unblinded $\mathrm{OC}$ as the standard of reference. Sensitivities and specificities were calculated for each single test, and for combinations of FS and stool tests. CTC radiation exposure was measured, and patient comfort levels and preferences were assessed by questionnaire.

Results: 221 adenomas were detected in 307 subjects who completed CTC (mean radiation dose, $4.5 \mathrm{mSv}$ ) and OC; 269 patients provided stool samples for both FOBT and FIT. Sensitivities of OC, CTC, FS, FIT and FOBT for advanced colonic neoplasia were 100\% (95\% Cl 88.4\% to $100 \%$ ), $96.7 \%$ (82.8\% to $99.9 \%$ ), $83.3 \%$ (95\% Cl $65.3 \%$ to $94.4 \%), 32 \%(95 \% \mathrm{Cl} 14.9 \%$ to 53.5$)$ and $20 \%(95 \% \mathrm{Cl}$ $6.8 \%$ to $40.7 \%)$, respectively. Combination of FS with FOBT or FIT led to no relevant increase in sensitivity. 12 of 45 advanced adenomas were smaller than $10 \mathrm{~mm}$. 46\% of patients preferred CTC and 37\% preferred OC $(p<0.001)$.

Conclusions: High-resolution and low-dose CTC is feasible for colorectal cancer screening and reaches sensitivities comparable with OC for polyps $>5 \mathrm{~mm}$. For patients who refuse full bowel preparation and OC or CTC, FS should be preferred over stool tests. However, in cases where stool tests are performed, FIT should be recommended rather than FOBT.

Colorectal cancer is one of the major public health issues in industrialised countries. Most colorectal cancers are thought to originate from benign adenomatous polyps that develop over a period of many years. ${ }^{1}$ Early detection followed by removal of adenomas has been shown to reduce incidence and colorectal cancer-related mortality. ${ }^{23}$ Therefore, screening of the asymptomatic and average risk population is recommended by many organisations and expert panels, and is reimbursed by insurance companies in several countries. ${ }^{4-7}$ Next to colonoscopy, flexible sigmoidoscopy (FS) and guaiac-based faecal occult blood test (FOBT) are widely applied screening procedures which have been compared prospectively with each other. Colonoscopy has been found to be the screening test with the highest sensitivity and outperforms FS and FOBT which miss a significant number of relevant adenomas. ${ }^{8}$ Colonoscopy, however, is not a perfect test in itself, and misses $6-12 \%$ of large adenomas. $^{9-11}$

CT colonography (CTC), also known as virtual colonoscopy, and faecal immunochemical tests (FITs) have been proposed as screening tests for colonic neoplasia. ${ }^{12-14}$ They have at present not been integrated into screening programmes. Based on recent research, CTC shows heterogeneous results in the detection of colonic polyps: some studies demonstrated high sensitivity in the detection of relevant colorectal adenomas, ${ }^{8} 131516$ while other trials showed less encouraging results with reported sensitivities of slightly more than $50 \% .{ }^{17}{ }^{18}$ Another important issue of CTC is the theoretical cancer risk associated with the radiation exposure. ${ }^{12} 19$ Therefore, if this test were to be acceptable as a mass screening instrument, radiation exposure of a single examination must be kept low, repeated examinations need to be avoided and sensitivity for relevant lesions must be high. Standard CTC will result in radiation doses of 10-12 mSv. Smaller series operating 4-slice scanners with low-dose protocols have reported effective doses of $2.1-7.8 \mathrm{mSv}^{15}{ }^{20}$ With 64 multidetector row CTC, increases in dose have been observed in different anatomical regions. ${ }^{21}$ Recently, a protocol that employs an online dose modulation algorithm that will lead to a $35 \%$ decrease in radiation exposure at preserved image quality was developed. ${ }^{22}$

Advanced colonic neoplasia comprises the entities invasive cancer and advanced adenoma. Advanced adenoma is defined as a lesion of adenomatous histology that meets at least one of the following criteria: a size of $\geqslant 10 \mathrm{~mm}$, the presence of a villous component of at least $25 \%$ or the presence of high-grade dysplasia. ${ }^{23}$ As these benign lesion are associated with a relatively high risk of progression to cancer, their removal effectively disrupts the adenoma-carcinoma pathway that is believed to be responsible for the majority of colorectal cancers. ${ }^{24}$ The prevalence of advanced adenoma in a screening population lies 
within a range of $3.7-15 \%$ and the prevalence of cancer has been reported to be $0.9 \%$ (range $0.5-1.3 \%$ ). ${ }^{8325}$ While it is not debated that adenoma larger than $1 \mathrm{~cm}$ and cancer need to be detected by a screening test, the relevance and handling of diminutive $(\leqslant 5 \mathrm{~mm})$ and small $(6-9 \mathrm{~mm})$ adenomas detected by CTC screening is currently under discussion. Recently, surveillance of polyps of 6-9 $\mathrm{mm}$ in diameter and non-reporting of diminutive lesions has been advocated. ${ }^{8}{ }^{13} 26$ Up to now, there are no data to support that this strategy would lead to an increase in carcinoma incidence in a screening population. However, the prevalence of advanced adenoma in small lesions is about $5 \%$, and the prevalence of cancer in this size group has been reported to be $0.1 \%{ }^{27}{ }^{28}$

We undertook this study to compare prospectively the performance of the three most commonly applied colorectal cancer screening tests, optical colonoscopy (OC), FS and FOBT, with high-resolution, low-dose CTC and FIT. For the first time, five different screening tests were compared in the same patients. CTC examinations were exclusively carried out using a 64-dector row scanner employing a low-dose protocol. We report the sensitivity, specificity, and positive and negative predictive values for the detection of patients with advanced adenoma and adenoma of various sizes for each test. We analysed the performance according to the polyp of highresolution CTC as a screening instrument compared with OC.

\section{METHODS}

\section{Study subjects}

The study protocol of this prospective colorectal cancer screening cohort study was approved by the institutional ethical committee, and the study meets all criteria put forth by the Declaration of Helsinki. Participants had to be $>50$ years of age and free of symptoms of colonic diseases such as melaenic stools, haematochezia, diarrhoea, relevant changes in stool frequency or abdominal pain. Exclusion criteria also included prior $\mathrm{OC}$ within the last 5 years, and positive family history for colorectal cancer (one first-degree relative diagnosed with CRC before age 60 or two first-degree relatives diagnosed with CRC at any age). Persons with a history of or present inflammatory bowel disease, hereditary colorectal cancer syndromes, a body weight $>150 \mathrm{~kg}$ or severe cardiovascular or pulmonary disease were also excluded. All participants provided written informed consent before their participation in the trial.

\section{Study procedure}

For each enrolled patient, a detailed medical history was taken prior to CTC. Patients also completed a questionnaire regarding their personal and family medical history. Patient comfort levels were assessed before and after CTC as well as after OC using standardised questionnaires. Study participants were asked to rate the discomfort related to bowel preparation, CTC and OC on a 6 -point scale. Complaints were rated as $1=$ none, $2=$ very mild, $3=$ mild, $4=$ moderate, $5=$ severe, and $6=$ unbearable.

\section{Bowel preparation}

A package including instructions and medication for bowel purgation, three FOBT slides and two $10 \mathrm{ml}$ stool sample containers for FIT was mailed to the participants. Before initiation of bowel lavage, FOBT samples were taken on three consecutive days. The two stool samples for FIT were collected from two different parts from the same stool and stored refrigerated. Bowel preparation was based on a standard "wet prep" regimen including 4 litres of polyethylene glycol solution
(KleanPrep, Norgine Pharmaceuticals, Marburg, Germany) and a commercially available combination of four tablets $(5 \mathrm{mg}$ each, for a total of $20 \mathrm{mg}$ ) of bisacodyl and $30 \mathrm{ml}$ of sodium phosphate (Prepacol, Guerbet Pharma, Sulzbach, Germany). Study participants were asked to follow a clear liquid diet from $12: 00 \mathrm{~h}$ the day before the examinations and ingest the bisacodyl tablets as well as the sodium phosphate solution at 14:00 h, and drink 3 litres of polyethylene glycol (PEG) between 17:00 $\mathrm{h}$ and 20:000 $\mathrm{h}$ on the day before CTC/OC. The last litre of PEG was drunk in the morning before examinations. To this last litre of $\mathrm{PEG}, 50 \mathrm{ml}$ of the iodinated contrast agent iopamidol (Solutrast 300, BraccoAltana Pharma, Milan, Italy) were added in order to tag residual fluid.

\section{CT colonography}

CTC scans were performed on a 64-channel multidetector row scanner (Siemens Somatom Sensation 64, Siemens Medical Solutions, Forchheim, Germany) at a collimation of $0.6 \mathrm{~mm}$ for high-resolution scanning. Images were reconstructed using a standard soft tissue kernel at a slice thickness of $0.75 \mathrm{~mm}$ and $0.5 \mathrm{~mm}$ reconstruction increment. Tube voltage was $120 \mathrm{kVp}$, and tube current-time product reference values were $70 \mathrm{mAs}$ in the supine and $30 \mathrm{mAs}$ in the prone position. An online dose modulation technique (Care Dose 4D, Siemens Medical Solutions) was used to adapt the tube current automatically to patient anatomy, ${ }^{22}$ and dose-length products were recorded for calculation of radiation exposure. Effective patient doses were calculated using appropriately normalised coefficients. ${ }^{29}$ No intravenous contrast agent was administered. A $20 \mathrm{mg}$ aliquot of $n$-butyl scopolamine (Buscopan, Boeringer Ingelheim Pharmaceuticals, Ingelheim, Germany) was administered intravenously for bowel relaxation.

Patients were positioned on the scanner table in the right decubitus position and bowel distension was achieved after placement of a rectal tube by manual air insufflation $(n=80)$ or automated $\mathrm{CO}_{2}$ application $(\mathrm{n}=227)$ using a commercially available insufflator (Protocol, E-Z-EM, Lake Success, New York, USA). Adequacy of colonic distension was determined by a radiologist on the CT scout film of the abdomen. Subsequently, the first set of images was obtained in a 7-9 s breath-hold with the patient in the supine position. After repositioning, the prone data set was obtained. Data sets were automatically sent to a 3D workstation (Syngo Workplace 2006 version VB 30, Siemens Medical Solutions). All scans were read by one of three experienced abdominal radiologists who had read $>300$ CTC examinations prior to the study using a primary $3 \mathrm{D}$ approach with $2 \mathrm{D}$ for problem solving. Immediately after CTC, patients were transferred to the endoscopy suite.

\section{Optical colonoscopy and flexible sigmoidoscopy}

Video colonoscopy was performed by one of six experienced gastroenterologists who had performed $>1000$ colonoscopies each before the start of the trial using video endoscopy (CF-O 160 series, Olympus Medical Systems, Hamburg, Germany). If desired, Disoprivan (Propofol, B. Braun Melsungen AG, Germany) was administered intravenously. Lesions were measured by comparison of their size with an open biopsy forceps. All polyps were resected or biopsied and retrieved at colonoscopy, and sent to histopathology for analysis. Sigmoidoscopy was defined as endoscopic examination of the rectum and sigmoid colon. FS results were deduced from OC results; no separate endoscopy was performed. 
Table 1 Distribution of adenomas and non-adenomatous polyps in 307 asymptomatic adults

\begin{tabular}{|c|c|c|c|c|}
\hline & \multicolumn{4}{|c|}{ Polyp size } \\
\hline & $<\mathbf{6 ~ m m}$ & 6-9 mm & $>9 \mathrm{~mm}$ & All \\
\hline \multicolumn{5}{|l|}{ Rectum } \\
\hline Adenomatous & 5 & 4 & 5 & 14 \\
\hline Non-adenomatous & 84 & 6 & 1 & 91 \\
\hline \multicolumn{5}{|l|}{ Sigmoid colon } \\
\hline Adenomatous & 33 & 16 & 15 & 64 \\
\hline Non-adenomatous & 78 & 1 & 0 & 79 \\
\hline \multicolumn{5}{|l|}{ Descending colon } \\
\hline Adenomatous & 24 & 6 & 4 & 34 \\
\hline Non-adenomatous & 26 & 2 & 0 & 28 \\
\hline \multicolumn{5}{|l|}{ Transverse colon } \\
\hline Adenomatous & 22 & 4 & 4 & 30 \\
\hline Non-adenomatous & 36 & 3 & 1 & 40 \\
\hline \multicolumn{5}{|l|}{ Ascending colon } \\
\hline Adenomatous & 41 & 9 & 2 & 52 \\
\hline Non-adenomatous & 23 & 2 & 1 & 26 \\
\hline \multicolumn{5}{|l|}{ Caecum } \\
\hline Adenomatous & 22 & 2 & 3 & 27 \\
\hline Non-adenomatous & 24 & 1 & 1 & 26 \\
\hline \multicolumn{5}{|l|}{ All segments } \\
\hline Adenomatous & 147 & 41 & 33 & 221 \\
\hline Non-adenomatous & 271 & 15 & 4 & 290 \\
\hline
\end{tabular}

\section{Documentation and matching of findings}

All findings were documented on a standardised report form. For each of six colonic segments (caecum, ascending colon, transverse colon, descending colon, sigmoid colon and rectum), the absence or presence of polyps was determined and lesion sizes were coded as diminutive $(\leqslant 5 \mathrm{~mm})$, small $(6-9 \mathrm{~mm})$ or large $(\geqslant 10 \mathrm{~mm})$. These size categories were based on previous research and the consensus statements of the European Society of Gastrointestinal and Abdominal Radiology and the Working Group on Virtual Colonoscopy. ${ }^{26} 30$ In the endoscopy suite, the report form containing the CTC results was provided to one of the endoscopy nurses who revealed the results to the endoscopist after withdrawal of the endoscope from each colonic segment. This technique, known as "segmental unblinding", allows for exact correlation of CTC and OC findings and can therefore be considered an enhanced gold standard. In the case of a discrepancy between CTC and OC first-look findings, an immediate colonscopic re-examination ("second look") of the respective colonic segment has to be performed; ${ }^{13} 17$ if results were discrepant after the second look, the radiologist was contacted and described the exact localisation of the lesion to the endoscopist who subsequently reexamined the segment. First- and second-look detections at OC were documented separately. In the case of concordance of CTC and OC findings, no second-look examination was performed. A lesion was rated a true positive detection if colonoscopy and CTC detected a polyp in the same or adjacent segment of the colon, and if the measured size of the lesion was within the same size category or if there was a deviation of no more than one size category. ${ }^{13}$ Only polyps detected in the rectum and sigmoid colon were included for analysis of the performance of flexible sigmoidoscopy. ${ }^{4}$

\section{Stool tests}

FOBTs were performed immediately, and stool samples were deep frozen at $-80^{\circ} \mathrm{C}$. The FOBT was judged to be positive if one of the three samples per patient yielded a positive test reaction.

For FIT, the provided stool samples were extracted by means of a stool sampling device (Sentinel Diagnostics, Milan, Italy) shaped like a standard analyser test tube filled with haemoglobin $(\mathrm{Hb})$ extracting buffer solution. The sample probe of the device has a serrated tip, which was poked into the stool at three different positions and pushed back into the tube through a tight membrane, removing most of the excess stool, leaving a quantitative amount of $10 \mathrm{mg}$ of stool in the serrations. Test reproducibility of quantitative stool transfer was shown to be $6.1 \%$ at a $\mathrm{Hb}$ mean concentration of $198 \mathrm{ng} / \mathrm{ml}$ and $5.2 \%$ at a $\mathrm{Hb}$ mean concentration of $600 \mathrm{ng} / \mathrm{ml}$ (homogenised stool sample, 11 different positions, two replicates each). After 30 min of mixing on a head-over-head rotator, the sampling device was de-capped, transferred onto an Architect c8000 Clinical Chemistry Analyzer (Abbott Diagnostics, Abbott Park, Illinois, USA) and $\mathrm{Hb}$ concentration measured by means of the FOB Gold immunoturbitrimetric latex assay (Sentinel Diagnostics, Milan, Italy). The FOB Gold assay is based on the antigen-antibody agglutination between human $\mathrm{Hb}$ in the sample and polyclonal anti-human $\mathrm{Hb}$ antibodies absorbed on polystyrene particles. Agglutination is measured as an increase in absorbance at $570 \mathrm{~nm}$ compared with a standard calibration curve and is proportional to the concentration of human $\mathrm{Hb}$ in the sample. Between-run confidence values were $5.6 \%$ at a $\mathrm{Hb}$ mean concentration of $80.3 \mathrm{ng} / \mathrm{ml}$ and $4.6 \%$ at a $\mathrm{Hb}$ mean concentration of $304 \mathrm{ng} / \mathrm{ml}$, respectively. The FIT was performed in each of the two samples per patient, and the higher value among these two entered the calculation. The lowest detection limit was $14 \mathrm{ng} / \mathrm{ml}$, which corresponds to the cut-off value for the calculations for specificity and sensitivity.

\section{Statistical analysis}

Prior to commencement of the trial, we performed statistical analyses to determine the required population size. These were based on the expected prevalence of colonic adenomas in an asymptomatic European population. Our statistician determined the number of individuals to be screened by precision of the $95 \%$ CI using normal approximations of binomial distributions. The study was powered to detect a $10 \%$ difference in OC and CTC sensitivity for detection of polyps $>5 \mathrm{~mm}$, and the number of patients to be screened was determined to be 300. All data were entered into a database and calculations were done using SAS Statistical Software Version 9.1 (SAS Institute Inc., Cary, North Carolina, USA). Sensitivities and specificities were calculated for OC and CTC on a per-polyp basis for advanced adenomas and for any polyp histology at cut-off sizes of 5 and $9 \mathrm{~mm}$. Per-patient sensitivities and specificities were calculated for all tests at size thresholds of 5 and $9 \mathrm{~mm}$. Specificities, and positive and negative predictive values were calculated for all tests.

\section{RESULTS}

A total of 311 consecutively enrolled asymptomatic adults underwent same-day CTC and OC (171 men and 140 women, 50-81 years of age, mean (SD) 60.5 (7.0) years). Four persons had to be excluded because of withdrawal from the trial after CTC $(n=2)$ or incomplete colonoscopy $(n=2)$. Stool samples for FIT were available in 285 persons, and FOBT slides were available in 276. Based on an interview prior to inclusion, all patients were considered to be at average risk. There were no clinically relevant complications due to OC or CTC. A total of 
Table 2 Characteristics of patients with advanced colonic neoplasia

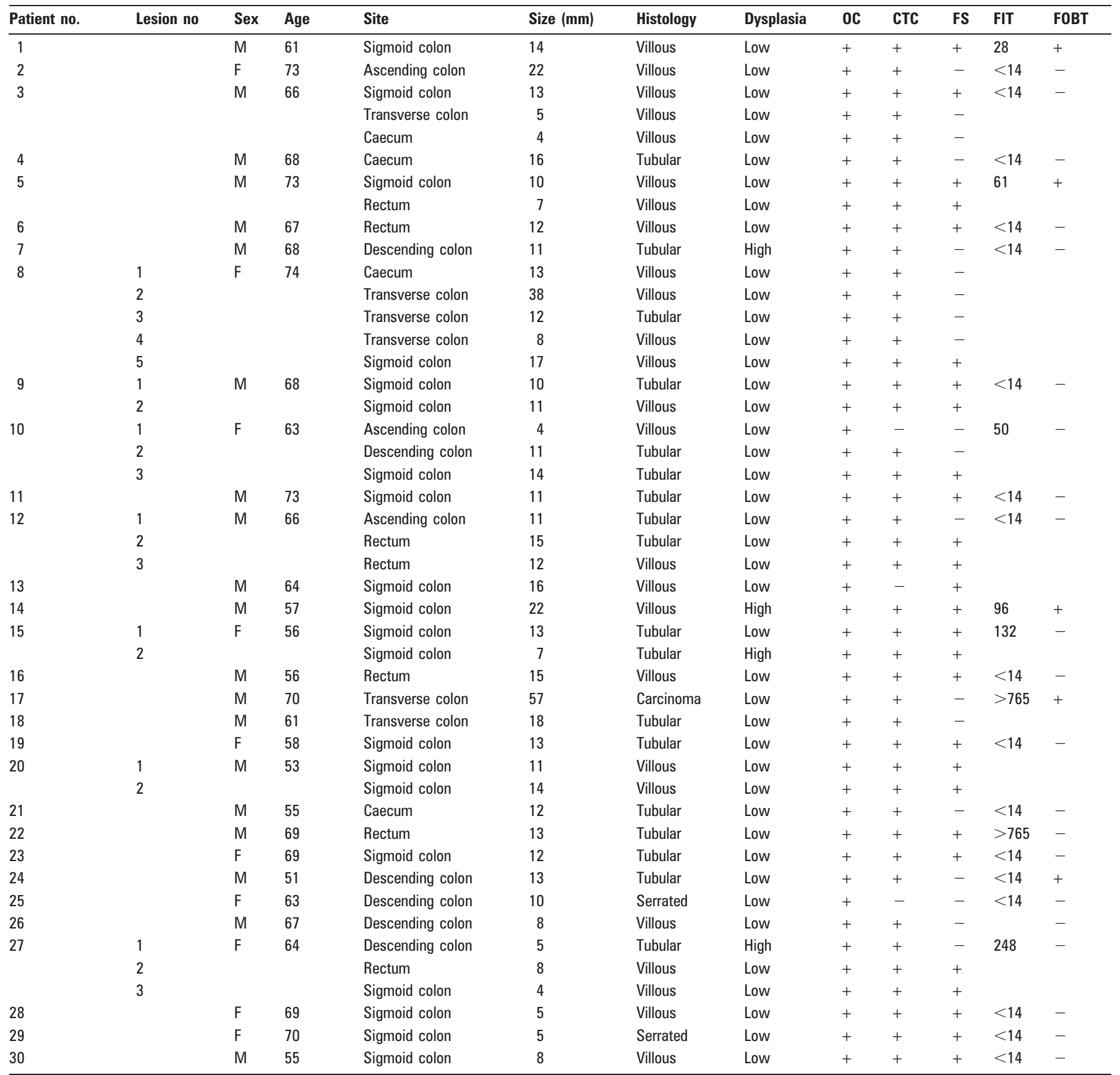

CTC, CT colonography; FIT, faecal immunochemical stool testing; FOBT, faecal occult blood testing; FS, flexible sigmoidoscopy; OC optical colonoscopy.

168 persons $(54.7 \%)$ chose sedation for colonoscopy. Mean radiation dose for CTC was $4.5(0.6) \mathrm{mSv}$ (range 3.5-6.1 $\mathrm{mSv}$ ). The supine scan at a reference $70 \mathrm{mAs}$ contributed a mean of $3.2 \mathrm{mSv}$, and the prone scan a mean of $1.3 \mathrm{mSv}$.

Table 1 summarises the distribution of polyps according to size and location. A total of 1842 colonic segments were analysed in 307 patients. Based on the gold standard (segmentally unblinded OC), 511 lesions were detected, 221 (43.2\%) were adenomatous and 290 (56.8\%) non-adenomatous. At least one adenoma of any size was detected in 113 participants (36.8\%). The maximum number of polyps detected in one participant was nine. A total of 418 (81.8\%) polyps were $\leqslant 5 \mathrm{~mm}, 56(11.0 \%)$ polyps measured $6-9 \mathrm{~mm}$, and $37(7.2 \%)$ polyps were $>9 \mathrm{~mm}$. A total of 248 polyps $(48.6 \%$; 78 adenomatous and 170 non-adenomatous polyps) were located within the reach of FS. Forty-six advanced lesions were detected: 7 lesions measuring $\leqslant 5 \mathrm{~mm}, 6$ lesions measuring 6$9 \mathrm{~mm}$, and 33 lesions measuring at least $10 \mathrm{~mm}$, including one stage T3 carcinoma of the transverse colon. The characteristics of patients with advanced adenoma are shown in table 2 .

Table 3 summarises the performance characteristics of the different methods for detection of adenomas according to the patient. OC reached the highest sensitivities for patients with adenomas of all size categories and identified $97.3 \%$ of patients with adenoma of all sizes, $97.8 \%$ of patients with adenomas $\geqslant 6 \mathrm{~mm}$ and all patients with adenomas $\geqslant 10 \mathrm{~mm}$. With sensitivities for identifying patients with adenomas $\geqslant 6 \mathrm{~mm}$ and $\geqslant 10 \mathrm{~mm}$ of $91.3 \%$ and $92 \%$, respectively, CTC reflected the excellent performance data reported recently. ${ }^{813}$ An example of a sessile lesion of $>10 \mathrm{~mm}$ in size seen at CTC and OC is shown 
in fig 1. In contrast, sigmoidoscopy, FIT and FOBT only identified $68,33.3$ and $23.8 \%$ of patients with adenomas $\geqslant 10 \mathrm{~mm}$. All tests except sigmoidoscopy identified a stage T3 colorectal cancer in the transverse colon of a 72-year-old man. Combination of sigmoidoscopy with FOBT or FIT resulted in increased detection rates for large adenomas of $76.2 \%$ and $71.4 \%$, respectively, compared with FS alone (68\%). A total of 269 patients had both stool tests, while only one of the tests was available in 38 patients; analysis of the group of 269 patients who had all tests did not differ significantly from the analysis including all 307 patients.

We detected 147 adenomas $\leqslant 5 \mathrm{~mm}$ and 41 adenomas 6-9 $\mathrm{mm}$ in size. Thirteen of these small adenomas were of advanced histology. The other $33(72 \%)$ advanced adenomas were $\geqslant 10 \mathrm{~mm}$ in size. OC identified $100 \%$ and CTC $96.7 \%$ of patients with advanced colonic neoplasia. The specificities of both methods equalled each other and resulted in similar positive and negative predictive values (table 3 ). The sensitivity according to the patient of sigmoidoscopy was higher for advanced lesions (25/30 patients, $83.3 \%$ ) than for adenoma $\geqslant 10 \mathrm{~mm}$ (17/25 patients, $68.0 \%$ ), reflecting the higher likelihood of advanced lesions in the rectosigmoid (27 of 46) compared with the rest of the colon (19 of 46). The sensitivities of FOBT and FIT for advanced lesions and adenoma $\geqslant 10 \mathrm{~mm}$ were not significantly different. Therefore, combination of sigmoidoscopy with stool tests did not increase sensitivity for advanced colonic neoplasia.

An analysis according to the polyp was performed for colonoscopy and CTC (table 4). The sensitivities for adenomas of all sizes was much higher for colonoscopy, with 212 of 221
(95.9\%) lesions detected compared with 155 adenomas (70.1\%) detected by CTC. This is mainly due to the comparably low performance of CTC in the diminutive size group. CTC only detected $59.2 \%$ of diminutive but $90.2 \%$ of $6-9 \mathrm{~mm}$ adenomas compared with colonoscopy, which detected $94.6 \%$ and $92.7 \%$. In contrast, CTC detected 31 of $33(93.9 \%)$ lesions in the large adenoma group and 43 of $46(93.5 \%)$ lesions in the advanced colonic neoplasia group. This was comparable with the detection rates of colonoscopy with sensitivities of $100 \%$ and $97.8 \%$, respectively, in the two categories. CTC had a higher sensitivity for small adenomas with advanced histology. While sensitivity of CTC for adenomas $<10 \mathrm{~mm}$ was only $66 \%$, CTC missed only one adenoma of those with advanced histology in this size group, resulting in a sensitivity of $93.5 \%$ for advanced colonic neoplasia of all sizes, which equals the sensitivity of colonoscopy.

In addition to identifying all patients with advanced colonic neoplasia an ideal colorectal cancer screening test would also be negative in all unaffected individuals. CTC had a per-patient specificity for polyps $\geqslant 6 \mathrm{~mm}$ of $93.1 \%$ and a specificity for patients without a polyp $\geqslant 10 \mathrm{~mm}$ of $97.9 \%$. The specificity of FOBT and FIT was $89.8 \%$ and $88.2 \%$, respectively.

A total of 256 patients (83.4\%) returned questionnaires for analysis, and 114 of these had sedation for OC. Regarding patient comfort, there was no difference between CTC and OC: $214(83.6 \%)$ patients rated discomfort at CTC as "absent", "very mild" or "mild", and 210 (82.0)\% chose these categories for OC. Thirty-seven per cent preferred OC for future screening, $46 \%$ CTC $(p<0.001)$, and $17 \%$ had no preference.

Table 3 Performance characteristics of OC, CTC, FS, FOBT and FIT in the detection of colonic adenomas in asymptomatic adults.

\begin{tabular}{|c|c|c|c|c|c|}
\hline & & All sizes & $>5 \mathrm{~mm}$ & $>9 \mathrm{~mm}$ & Advanced colonic neoplasia \\
\hline & & No./total no. $(\%(95 \% \mathrm{CI}))$ & No./total no. $(\%(95 \% \mathrm{CI}))$ & No./total no. $(\%(95 \%$ CI $))$ & No./total no. $(\%(95 \% \mathrm{CI}))$ \\
\hline \multirow[t]{4}{*}{$\mathrm{OC}$} & Sens & $110 / 113$ (97.3 (92.4 to 99.4$))$ & $45 / 46(97.8(88.5$ to 99.9$))$ & $25 / 25(100.0(86.3$ to 100.0$))$ & $30 / 30(100(88.4$ to 100$))$ \\
\hline & Spec & $116 / 194(59.8$ (52.5 to 66.8$))$ & $250 / 261$ (95.8 (92.6 to 97.9$))$ & $278 / 282(98.6$ (96.4 to 99.6$))$ & $119 / 277(43.0(37.1$ to 49$))$ \\
\hline & PPV & $110 / 188(58.5(51.1$ to 65.6$))$ & $45 / 56(80.4(67.6$ to 89.8$))$ & $25 / 29(86.2(68.3$ to 96.1$))$ & $30 / 188(16.0(11.0$ to 22.0$))$ \\
\hline & NPV & $116 / 119$ (97.5 (92.8 to 99.5$))$ & $250 / 251(99.6(97.8$ to 100$))$ & $278 / 278(100.0(98.7$ to 100.0$))$ & $119 / 119(100(96.9$ to 100$))$ \\
\hline \multirow[t]{2}{*}{ FS } & Sens & $81 / 113(71.7$ (62.4 to 79.8$))$ & $31 / 46(67.4(52.0$ to 80.5$))$ & $17 / 25(68.0(46.5$ to 85.1$))$ & $25 / 30(83.3(65.3$ to 94.4$))$ \\
\hline & NPV & $138 / 170(81.2(74.5$ to 86.8$))$ & $258 / 273(94.5$ (91.1 to 96.9$))$ & $281 / 289$ (97.2 (94.6 to 98.8$))$ & $165 / 170(97.1(93.3$ to 99$))$ \\
\hline \multirow[t]{4}{*}{ CTC } & Sens & $95 / 113(84.1$ (76.0 to 90.3$))$ & $42 / 46(91.3$ (79.2 to 97.6$))$ & $23 / 25(92.0(74.0$ to 99.0$))$ & $29 / 30(96.7(82.8$ to 99.9$))$ \\
\hline & Spec & $92 / 194(47.4(40.2$ to 54.7$))$ & $243 / 261$ (93.1 (89.3 to 95.9$))$ & $276 / 282(97.9$ (95.4 to 99.2$))$ & $109 / 277(39.4(33.6$ to 45.4$))$ \\
\hline & PPV & $95 / 197(48.2(41.1$ to 55.4$))$ & $42 / 60(70.0$ (56.8 to 81.2$))$ & 23/29 (79.3 (60.3 to 92.0$))$ & $29 / 197(14.7(10.1$ to 20.5$))$ \\
\hline & NPV & $92 / 110(83.6(75.4$ to 90.0$))$ & $243 / 247$ (98.4 (95.9 to 99.6$))$ & $276 / 278$ (99.3 (97.4 to 99.0$))$ & $109 / 110(99.1(95.0$ to 100$))$ \\
\hline FOBT & Sens & $20 / 99$ (20.2 (12.8 to 29.5$))$ & $7 / 40(17.5$ (7.3 to 32.8$))$ & $5 / 21(23.8(8.2$ to 47.2$))$ & $5 / 25(20.0(6.8$ to 40.7$))$ \\
\hline \multirow{3}{*}{ FIT } & Spec & $163 / 183(89.1$ (83.6 to 93.2$))$ & $216 / 245$ (88.2 (83.4 to 91.9$))$ & $226 / 264(85.6$ (80.8 to 89.6$))$ & $223 / 260(85.8$ (80.9 to 89.8$))$ \\
\hline & PPV & $25 / 45(55.6$ (40.0 to 70.4$))$ & $16 / 45(35.6(21.9$ to 51.2$))$ & $7 / 45$ (15.6 (6.5 to 29.5$))$ & $8 / 45(17.8$ (8.0 to 32.1$))$ \\
\hline & NPV & $163 / 240(67.9(61.6$ to 73.8$))$ & $216 / 240(90.0(85.5$ to 93.5$))$ & $226 / 240(94.2$ (90.4 to 96.8$))$ & $223 / 240(92.9$ (88.9 to 95.8$))$ \\
\hline \multirow[t]{4}{*}{$\mathrm{FS}+\mathrm{FOBT}$} & Sens & 71/99 (71.7 (61.8 to 80.3$))$ & $28 / 40(70.0(53.5$ to 83.4$))$ & $16 / 21(76.2$ (52.8 to 91.8$))$ & $20 / 25(80.0$ (59.3 to 93.2$))$ \\
\hline & Spec & $120 / 177(67.8(60.4$ to 74.6$))$ & $211 / 236(89.4$ (84.8 to 93.0$))$ & $228 / 255(89.4$ (85.0 to 92.9$))$ & $143 / 251(57.0(50.6$ to 63.2$))$ \\
\hline & PPV & $71 / 128$ (55.5 (46.4 to 64.3$))$ & $28 / 53(52.8$ (38.6 to 66.7$))$ & $16 / 43(37.2(23.0$ to 53.3$))$ & $20 / 128$ (15.6 (9.8 to 23.1$))$ \\
\hline & NPV & $120 / 148(81.1$ (73.8 to 87.0$))$ & $211 / 223$ (94.6 (90.8 to 97.2$))$ & $228 / 233$ (97.9 (95.1 to 99.3$))$ & $143 / 148$ (96.6 (92.3 to 98.9$))$ \\
\hline \multirow[t]{4}{*}{$\mathrm{FS}+\mathrm{FIT}$} & Sens & $79 / 102(77.5(68.1$ to 85.1$))$ & $32 / 40(80.0(64.4$ to 90.9$))$ & $15 / 21(71.4(47.8$ to 88.7$))$ & $21 / 25(84.0(63.9$ to 95.5$))$ \\
\hline & Spec & $119 / 183(65.0(57.6$ to 71.9$))$ & $215 / 245(87.8(83.0$ to 91.6$))$ & $225 / 264(85.2(80.4$ to 89.3$))$ & $138 / 260(53.1$ (46.8 to 59.3$))$ \\
\hline & PPV & $79 / 143(55.2(46.7$ to 63.6$))$ & $32 / 62(51.6$ (38.6 to 64.5$))$ & $15 / 54(27.8(16.5$ to 41.6$))$ & $21 / 143(14.7$ (9.3 to 21.6$))$ \\
\hline & NPV & $119 / 142(83.8(76.7$ to 89.4$))$ & $215 / 223(96.4(93.1$ to 98.4$))$ & $225 / 231$ (97.4(94.4 to 99.0$))$ & $138 / 142(97.2$ (92.9 to 99.2$))$ \\
\hline
\end{tabular}

Analysis according to the patient.

CTC, CT colonography; FIT, faecal immunochemical stool testing; FOBT, faecal occult blood testing; FS, flexible sigmoidoscopy; NPV, negative predictive value; OC optical colonoscopy; PPV, positive predictive value; Sens, sensitivity; Spec, specificity. 

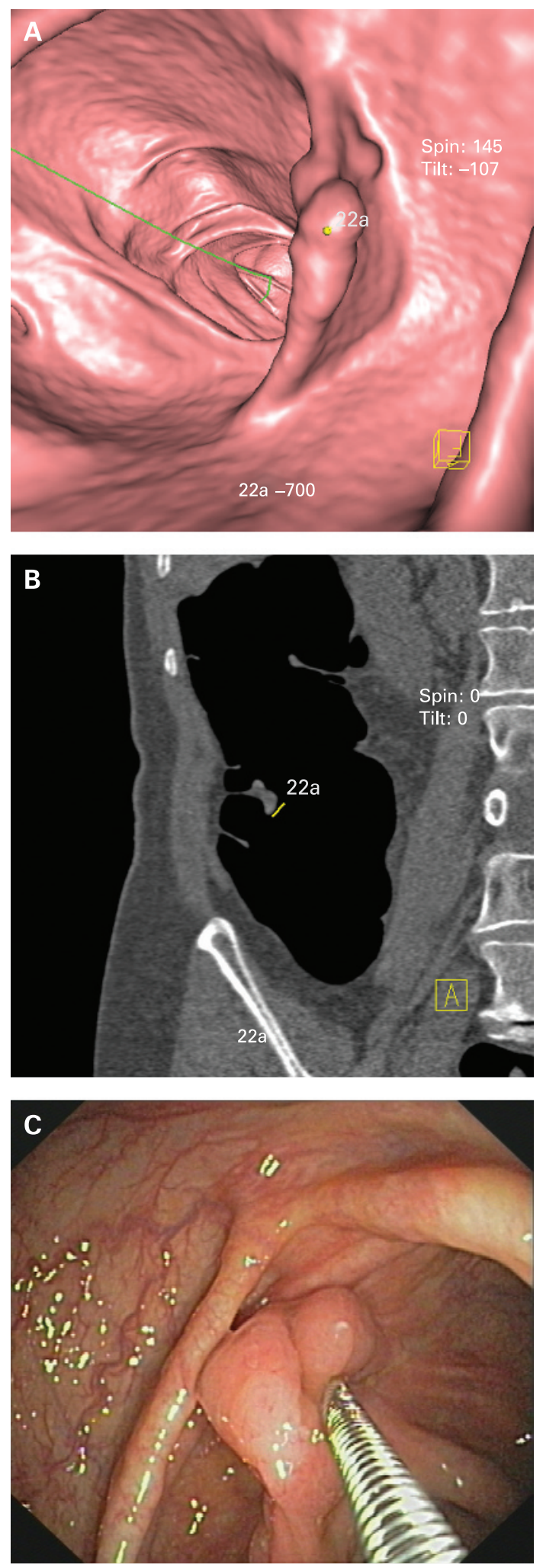

Figure 1 (A) High-resolution 3D CT colonography endoluminal view shows a $2.2 \mathrm{~cm}$ sessile polyp (marker "22a") in the ascending colon in a 72-year-old asymptomatic female. (B) Corresponding coronally reformatted CT image showing the same lesion. Isotropic data sets allow for reformation of images in any desired plane. (C) At colonoscopy, the lesion is confirmed. Histopathology revealed villous adenoma.

\section{DISCUSSION}

Colorectal cancer is believed to be largely preventable through effective screening. ${ }^{2}$ However, compliance with current screening recommendations is low and several predictors of nonadherence to screening colonoscopy have been identified. ${ }^{31-33} \mathrm{~A}$ major deterrent from screening is non-compliance with colonoscopy. Therefore, alternative strategies, including self-propelling and self-navigating colonoscopes, capsule colonoscopy, virtual colonography based on CT or MRI, and new generation stool tests based on immunological detection of blood or detection of DNA mutations, have been proposed and are at different stages of development. ${ }^{84}$ Prior to introduction, these methods need to be prospectively evaluated and compared with established tests.

There is good evidence that screening of asymptomatic persons with the use of FOBT or sigmoidoscopy can reduce mortality from colorectal cancer. ${ }^{23}{ }^{35} 36$ Several studies have analysed the combination of sigmoidoscopy with FOBT and found that combining the tests resulted in increased sensitivity for advanced neoplasia. ${ }^{24}{ }^{37}$ In our study, we deduced FS results from colonoscopy by determining the rectum and sigmoid colon as being accessible for this test. Population sensitivity of sigmoidoscopy for advanced adenoma was $83.3 \%$, which is in accordance with the above-mentioned studies. Our results for FS might have been improved by rigorous bowel preparation, which normally would not be employed for sigmoidoscopy. FOBT only detected $20 \%$ of advanced adenomas, and combination of FS with FOBT only resulted in an increase in the detection rate of large adenomas but not advanced adenomas. Immunochemical-based FITs detect human $\mathrm{Hb}$ in stool and have higher sensitivities for advanced colonic neoplasia than guaiac-based FOBT. ${ }^{14}{ }^{38}$ We found that FIT identified $32 \%$ of patients with advanced and $33.3 \%$ of patients with large adenomas, and the combination of FIT with FS resulted in only a slight increase in detection rates.

CTC is currently the most promising new screening method, and several studies have reported high sensitivities for adenomas. ${ }^{8}{ }^{13}$ Additionally, CTC has now for the first time been recommended for colorectal cancer screening by the American Cancer Society. ${ }^{39}$ We used a 64 -multidetector row CTC scanner that provides $0.4 \mathrm{~mm}$ isotropic resolution and employed $3 \mathrm{D}$ endoluminal CTC interpretation using a dedicated workstation. Reconstructing $0.75 \mathrm{~mm}$ slices leads to higher spatial resolution than in other trials published to date. This may have contributed to the high sensitivities for adenomas in our study. Interestingly, our CTC approach detected the majority of advanced adenomas $<10 \mathrm{~mm}$ in diameter. This may have been caused by the small number of advanced lesions in this size group; however, it has been postulated that adenomas in general are less deformable than non-neoplastic lesions, which leads to increased conspicuity at CTC. ${ }^{40} 41$

The relevance of diminutive and small polyps $1-9 \mathrm{~mm}$ in size has recently become a controversial topic. ${ }^{42}$ At least $20-30 \%$ of the average risk asymptomatic population above age 50 carry adenomatous polyps. ${ }^{43}$ The majority of these are $<10 \mathrm{~mm}$. However, controversy exists as to the likelihood that small adenomas harbour significant advanced histology or progress to colorectal cancer. This has important implications for reporting and follow-up. A recent consensus proposal for CTC reporting suggested that diminutive polyps do not need to be reported, and patients with $\leqslant 2$ polyps of $<10 \mathrm{~mm}$ are recommended to undergo follow-up CTC after 3 years rather than immediate colonoscopy for polypectomy, which is recommended for large polyps or if $\geqslant 3$ small polyps are present. ${ }^{26}$ As small and medium size lesions may contain advanced histology ${ }^{42}$ following this 
Table 4 Performance characteristics of OC and CTC in the detection of colonic adenomas

\begin{tabular}{|c|c|c|c|c|c|}
\hline & All sizes & $<6 \mathrm{~mm}$ & 6 to $9 \mathrm{~mm}$ & $>9 \mathrm{~mm}$ & Advanced colonic neoplasia \\
\hline & No./total no. $(\%(95 \% \mathrm{CI}))$ & No./total no. $(\%(95 \% \mathrm{CI}))$ & No./total no. $(\%(95 \% \mathrm{Cl}))$ & No./total no. $(\%(95 \% \mathrm{Cl}))$ & No./total no. $(\%(95 \% \mathrm{CI}))$ \\
\hline $\begin{array}{l}\text { Sensitivity } \\
\text { OC }\end{array}$ & $212 / 221(95.9(92.4$ to 98.1$))$ & $139 / 147(94.6$ (89.6 to 97.6$))$ & $38 / 41(92.7(80.1$ to 98.5$))$ & $33 / 33(100(89.4$ to 100$))$ & $45 / 46(97.8(88.5$ to 99.9$))$ \\
\hline $\begin{array}{l}\text { Sensitivity } \\
\text { CTC }\end{array}$ & $155 / 221(70.1$ (63.6 to 76.1$))$ & $87 / 147(59.2(50.8$ to 67.2$))$ & $37 / 41(90.2(76.9$ to 97.3$))$ & $31 / 33(93.9$ (79.8 to 99.3$))$ & $43 / 46(93.5(82.1$ to 98.6$))$ \\
\hline
\end{tabular}

Analysis according to polyp.

CTC, CT colonography; OC, optical colonoscopy.

recommendation might lead to an increase in colorectal cancer incidence and mortality. ${ }^{25}$

Another important issue is radiation exposure during CTC. Standard CTC techniques apply up to $12 \mathrm{mSv} .{ }^{12}{ }^{15}{ }^{44}$ It has been estimated that any amount of ionising radiation may lead to an increase in radiation-related cancer and death, and that up to $2 \%$ of cancers in the USA may be induced by diagnostic CT examinations. ${ }^{12} 19$ Therefore, medical imaging-associated radiation needs to be kept to a minimum especially in screening procedures. Using low-dose protocols and new dose modulation techniques, we were able to decrease the mean radiation dose to $4.5 \mathrm{mSv}$ for the entire examination. This value is significantly lower than the dose values reported in or calculated from other major trials that used spatial resolutions inferior to our protocol, and is lower than measured values for a 64-detector system without dose modulation techniques. ${ }^{45}$ Remarkably, image quality remained high even in the pelvis, an anatomical region that is prone to image noise-induced artefacts in CTC. ${ }^{22} 45$

Although more patients preferred CTC than OC for future screening (46\% vs $37 \%$ ), this preference was not as clear as in other comparative trials. Preferences were dependent on use of sedation for OC.

In conclusion, our results show that CTC performs equally as well as colonoscopy in detecting advanced adenomas. Therefore, future screening guidelines might include CTC as a primary screening test as an alternative to colonoscopy. Prerequisites for colorectal cancer screening by CTC are adequate training of radiologists, employment of high-resolution low-dose CT technique, and opportunity of same-day colonoscopy in the case of relevant findings in order to avoid repeat bowel preparation. FS should be preferred to stool tests in patients who refuse to undergo full bowel preparation or total colonoscopy. FIT has a higher sensitivity for adenomas than FOBT, but both stool tests are inferior to tests that allow visualisation of the colonic mucosa.

Acknowledgements: We appreciate the continuing support provided by the staff of the endoscopy and CT units during the duration of the study. We thank Roche Diagnostics for providing the FOBT tests, and E-Z-EM for providing the $\mathrm{CO}_{2}$ insufflator unit.

Competing interests: None.

Ethics approval: The study protocol was approved by the institutional ethical committee, and the study meets all criteria put forth by the Declaration of Helsinki.

\section{REFERENCES}

1. Mandel JS, Bond JH, Church TR, et al. Reducing mortality from colorectal cancer by screening for fecal occult blood. Minnesota Colon Cancer Control Study. N Engl J Med 1993;328:1365-71.

2. Winawer SJ, Zauber AG, Ho MN, et al. Prevention of colorectal cancer by colonoscopic polypectomy. The National Polyp Study Workgroup. N Engl J Med 1993;329:1977-81.

3. Mandel JS, Church TR, Bond JH, et al. The effect of fecal occult-blood screening on the incidence of colorectal cancer. N Engl J Med 2000;343:1603-7.

4. Lieberman DA, Weiss DG. One-time screening for colorectal cancer with combined fecal occult-blood testing and examination of the distal colon. N Engl J Med 2001;345:555-60.
5. Winawer SJ, Fletcher RH, Miller L, et al. Colorectal cancer screening: clinical guidelines and rationale. Gastroenterology 1997;112:594-642.

6. Rex DK. Current colorectal cancer screening strategies: overview and obstacles to implementation. Rev Gastroenterol Disord 2002;2(Suppl 1):S2-11.

7. Pickhardt PJ, Taylor AJ, Kim DH, et al. Screening for colorectal neoplasia with CT colonography: initial experience from the 1st year of coverage by third-party payers. Radiology 2006;241:417-25.

8. Kim DH, Pickhardt PJ, Taylor AJ, et al. CT colonography versus colonoscopy for the detection of advanced neoplasia. N Engl J Med 2007;357:1403-12.

9. Rex DK, Cutler CS, Lemmel GT, et al. Colonoscopic miss rates of adenomas determined by back-to-back colonoscopies. Gastroenterology 1997;112:24-8.

10. Pickhardt PJ, Nugent PA, Mysliwiec PA, et al. Location of adenomas missed by optical colonoscopy. Ann Intern Med 2004;141:352-9.

11. Heresbach D, Barrioz T, Lapalus MG, et al. Miss rate for colorectal neoplastic polyps: a prospective multicenter study of back-to-back video colonoscopies. Endoscopy 2008;40:284-90.

12. Brenner DJ, Georgsson MA. Mass screening with CT colonography: should the radiation exposure be of concern? Gastroenterology 2005;129:328-37.

13. Pickhardt PJ, Choi JR, Hwang I, et al. Computed tomographic virtual colonoscopy to screen for colorectal neoplasia in asymptomatic adults. N Engl J Med 2003;349:2191-200.

14. Guittet L, Bouvier V, Mariotte N, et al. Comparison of a guaiac based and an immunochemical faecal occult blood test in screening for colorectal cancer in a general average risk population. Gut 2007;56:210-4.

15. Macari M, Bini EJ, Xue X, et al. Colorectal neoplasms: prospective comparison of thin-section low-dose multi-detector row CT colonography and conventional colonoscopy for detection. Radiology 2002;224:383-92.

16. Yee J, Akerkar GA, Hung RK, et al. Colorectal neoplasia: performance characteristics of CT colonography for detection in 300 patients. Radiology 2001;219:685-92.

17. Cotton PB, Durkalski VL, Pineau BC, et al. Computed tomographic colonography (virtual colonoscopy): a multicenter comparison with standard colonoscopy for detection of colorectal neoplasia. JAMA 2004;291:1713-9.

18. Rockey DC, Paulson E, Niedzwiecki D, et al. Analysis of air contrast barium enema, computed tomographic colonography, and colonoscopy: prospective comparison. Lancet 2005;365:305-11.

19. Brenner DJ, Hall EJ. Computed tomography — an increasing source of radiation exposure. N Engl J Med 2007;357:2277-84.

20. Iannaccone R, Laghi A, Catalano C, et al. Detection of colorectal lesions: lower-dose multi-detector row helical CT colonography compared with conventional colonoscopy. Radiology 2003;229:775-81.

21. Dewey M, Hoffmann H, Hamm B. CT coronary angiography using 16 and 64 simultaneous detector rows: intraindividual comparison. Rofo 2007;179:581-6.

22. Graser A, Wintersperger BJ, Suess C, et al. Dose reduction and image quality in MDCT colonography using tube current modulation. AJR Am J Roentgenol 2006; 187:695-701.

23. Lieberman DA, Weiss DG, Bond JH, et al. Use of colonoscopy to screen asymptomatic adults for colorectal cancer. Veterans Affairs Cooperative Study Group 380. N Engl J Med 2000;343:162-8.

24. Bond JH. Doubling time of flat and polypoid colorectal neoplasms: defining the adenoma-carcinoma sequence. Am J Gastroenterol 2000;95:1621-3.

25. Hur C, Chung DC, Schoen RE, et al. The management of small polyps found by virtual colonoscopy: results of a decision analysis. Clin GastroenterolHepatol 2007:5:237-44.

26. Zalis ME, Barish MA, Choi JR, et al. CT colonography reporting and data system: a consensus proposal. Radiology 2005;236:3-9.

27. Yoo TW, Park DI, Kim YH, et al. Clinical significance of small colorectal adenoma less than $10 \mathrm{~mm}$ : the KASID study. Hepatogastroenterology 2007;54:418-21.

28. Moravec M, Lieberman D, Holub J, et al. Rate of advanced pathologic features in 6$9 \mathrm{~mm}$ polyps in patients referred for colonoscopy screening. Gastrointest Endosc 2007:65822.

29. European guidelines on quality criteria for computed tomography. 1999. http://www.drs.dk/guidelines/ct/quality/mainindex.htm (Accessed 4 Nov 2008)

30. Taylor SA, Halligan S, Slater A, et al. Polyp detection with CT colonography: primary $3 \mathrm{D}$ endoluminal analysis versus primary 2D transverse analysis with computerassisted reader software. Radiology 2006;239:759-67.

31. Harewood GC, Wiersema MJ, Melton LJ III. A prospective, controlled assessment of factors influencing acceptance of screening colonoscopy. Am J Gastroenterol 2002;97:3186-94. 
32. Greiner KA, Born W, Nollen N, et al. Knowledge and perceptions of colorectal cancer screening among urban African Americans. J Gen Intern Med 2005;20:97783.

33. Denberg TD, Melhado TV, Coombes JM, et al. Predictors of nonadherence to screening colonoscopy. J Gen Intern Med 2005;20:989-95.

34. Chen WD, Han ZJ, Skoletsky J, et al. Detection in fecal DNA of colon cancer-specific methylation of the nonexpressed vimentin gene. J Natl Cancer Inst 2005;97:112432.

35. Selby JV, Friedman GD, Quesenberry CP Jr, et al. A case-control study of screening sigmoidoscopy and mortality from colorectal cancer. N Engl J Med 1992;326:653-7.

36. Newcomb PA, Norfleet RG, Storer BE, et al. Screening sigmoidoscopy and colorectal cancer mortality. J Natl Cancer Inst 1992;84:1572-5.

37. Rasmussen M, Kronborg 0 , Fenger $C$, et al. Possible advantages and drawbacks of adding flexible sigmoidoscopy to hemoccult-II in screening for colorectal cancer. A randomized study. Scand J Gastroenterol 1999;34:73-8.

38. Smith A, Young GP, Cole SR, et al. Comparison of a brush-sampling fecal immunochemical test for hemoglobin with a sensitive guaiac-based fecal occult blood test in detection of colorectal neoplasia. Cancer 2006;107:2152-9.
39. Levin B, Lieberman DA, McFarland B, et al. Screening and surveillance for the early detection of colorectal cancer and adenomatous polyps, 2008: a joint guideline from the American Cancer Society, the US Multi-Society Task Force on Colorectal Cancer, and the American College of Radiology. Gastroenterology 2008;134:1570-95.

40. Bertoni G, Sassatelli R, Conigliaro R, et al. Visual "disappearing phenomenon" can reliably predict the nonadenomatous nature of rectal and rectosigmoid diminutive polyps at endoscopy. Gastrointest Endosc 1994;40:588-91.

41. Pickhardt PJ, Choi JR, Hwang I, et al. Nonadenomatous polyps at CT colonography: prevalence, size distribution, and detection rates. Radiology 2004;232:784-90.

42. Butterly LF, Chase MP, Pohl $\mathrm{H}$, et al. Prevalence of clinically important histology in small adenomas. Clin Gastroenterol Hepatol 2006;4:343-8.

43. Zauber AG, Winawer SJ. Initial management and follow-up surveillance of patients with colorectal adenomas. Gastroenterol Clin North Am 1997;26:85-101.

44. van Gelder RE, Nio CY, Florie J, et al. Computed tomographic colonography compared with colonoscopy in patients at increased risk for colorectal cancer. Gastroenterology 2004;127:41-8.

45. Luz $\mathbf{0}$, Buchgeister M, Klabunde $\mathrm{M}$, et al. Evaluation of dose exposure in 64-slice CT colonography. Eur Radiol 2007;17:2616-21. 


\section{Comparison of CT colonography,} colonoscopy, sigmoidoscopy and faecal occult blood tests for the detection of advanced adenoma in an average risk population

A Graser, P Stieber, D Nagel, et al.

Gut 2009 58: 241-248 originally published online October 13, 2008 doi: $10.1136 /$ gut.2008.156448

Updated information and services can be found at:

http://gut.bmj.com/content/58/2/241.full.html

\section{These include:}

References This article cites 43 articles, 10 of which can be accessed free at: http://gut.bmj.com/content/58/2/241.full.html\#ref-list-1

Article cited in:

http://gut.bmj.com/content/58/2/241.full.html\#related-urls

Email alerting Receive free email alerts when new articles cite this article. Sign up in service the box at the top right corner of the online article.

Topic Articles on similar topics can be found in the following collections Collections

Notes

To request permissions go to:

http://group.bmj.com/group/rights-licensing/permissions

To order reprints go to:

http://journals.bmj.com/cgi/reprintform

To subscribe to BMJ go to:

http://group.bmj.com/subscribe/ 